\title{
Parches invadidos por Ugni molinae en isla Robinson Crusoe: ¿Hay plantas nativas y endémicas capaces de vivir en ellos?
}

\section{Ugni molinae invaded patches in Robinson Crusoe Island: Are there native and endemic plants able to live within them?}

\author{
Diego Alarcón ${ }^{1,2 *}$, Patricio López-Sepúlveda ${ }^{1}$, Glenda Fuentes ${ }^{1}$, Hellen Montoya ${ }^{1}$, Patricio \\ Peñallillo ${ }^{3} \&$ Pedro Carrasco ${ }^{1}$
}

${ }^{1}$ Departamento de Botánica, Facultad de Ciencias Naturales y Oceanográficas, Casilla 160-C, Universidad de Concepción, Concepción, Chile.

${ }^{2}$ Instituto de Ecología y Biodiversidad, Las Palmeras 3425, Ñuñoa, Santiago, Chile.

${ }^{3}$ Instituto de Ciencias Biológicas, Universidad de Talca, Av. Lircay s/n, Talca, Chile.

*chilebosque@gmail.com

\begin{abstract}
It is unknown if any Juan Fernández native plants may tolerate the aggressive invasion of the introduced shrub Ugni molinae. We found sixteen native species growing within these highly invaded patches. The endemic shrub Gaultheria racemulosa (syn. Pernettya rigida) was the most important species according to vegetation cover in invaded patches, therefore relevant in future control management options.
\end{abstract}

La invasión de plantas alóctonas representa una de las mayores amenazas a la conservación de la biodiversidad en islas oceánicas (Greimler et al. 2002, Funk \& Vitousek 2007, Kawakami \& Okochi 2010, Lookwood et al. 2013, Smith-Ramírez et al. 2017). Éste es uno de los principales problemas existentes en el Archipiélago de Juan Fernández, Región de Valparaíso, Chile, en cuyas islas (Robinson Crusoe, Alejandro Selkirk y Santa Clara), numerosas especies introducidas se han transformado en invasoras, desplazando la vegetación nativa, con la mayoría de sus especies endémicas y amenazadas de extinción (Danton et al. 2006, Stuessy et al. 2018a, 2018b). Ugni molinae es un arbusto nativo de Chile continental y Argentina, alóctono en el Archipiélago de Juan Fernández, cuya introducción a las islas se presume anterior al año 1896 (Johow 1896), se ha transformado en una especie invasora, constituyéndose en una de las más complejas amenazas a la biodiversidad local, llegando a invadir una proporción del total de la isla Robinson Crusoe correspondiente a 4,6\%, según Dirnböck et al. (2003), o al 2,6\% según Díaz (2012) citado por SmithRamírez et al. (2017). La dispersión de sus semillas mediada por el ave zorzal (Turdus falcklandii), ha permitido una muy eficiente invasión (Smith-Ramírez et al. 2013). Hay poco conocimiento informado acerca de la dinámica de estos parches de vegetación luego de consolidada la invasión. Se ha asumido que se trata de unidades con amplio dominio de $U$. molinae y se desconoce si hay especies nativas y endémicas del bosque fernandeziano capaces de vivir bajo o entre esta agresiva invasora. Los antecedentes que se tienen sobre estos parches invadidos por U. molinae incluyen el efecto de desplazamiento de especies nativas y endémicas, que ocurre en aquellas laderas erosionadas o expuestas al viento, sobre una altitud mayor a $250 \mathrm{~m}$ s.n.m., afectando principalmente a poblaciones de Gaultheria racemulosa $[=$ Pernettya rigida], Ugni selkirkii y Blechnum cycadifolium (Greimler et al. 2002). Bastías (2014) informa de algunas especies asociadas a parches invadidos por $U$. molinae encontradas al momento de realizar ensayos de control, únicamente en sectores de altitudes más bajas (190 m s.n.m.), mencionando las especies Blechnum hastatum, Adiantum chilense, Rumohra berteroana, Erigeron fernandezius y al género Hymenophyllum.

El objetivo de este trabajo fue conocer si existen especies de flora nativa y/o endémica del Archipiélago de Juan Fernández que puedan vivir en las condiciones que genera la masiva invasión de $U$. molinae en sectores de mayor altitud en la Isla Robinson Crusoe, donde aún se conserva bosque nativo en la isla y es en esas zonas donde la invasora estaría afectando de mayor manera a las comunidades nativas. Para ello, se realizó un registro preliminar de la flora vascular y de la importancia relativa de las especies, tanto al interior de los parches invadidos como también en un rodal de bosque remanente fernandeziano aledaño. Este trabajo se desarrolló en el área denominada Salsipuedes, al noroeste de la localidad urbana de San Juan Bautista (ver Fig. 1) a altitudes entre los 400 y $500 \mathrm{~m}$ s.n.m.

Se localizaron 15 puntos seleccionados bajo los siguientes criterios: ser parches ampliamente dominados 
por $U$. molinae con cobertura superior a $75 \%$ de dicha especie invasora, con accesibilidad factible desde el sendero de Salsipuedes, considerando dificultad de la topografía local extrema y con presencia cercana de un parche de bosque nativo fernandeziano remanente. En la selección de las unidades se consideró completar un gradiente de distancia al parche de bosque nativo y conjuntamente incluir un gradiente de altitud. La naturaleza del terreno de topografía abrupta dificultó realizar un intervalo regular entre los puntos. No obstante, la distancia media entre pares de puntos más cercanos fue de $23 \mathrm{~m}$, con una desviación estándar de 16,2 m, localización que se puede apreciar en la Fig. 1. Tanto en la unidad de bosque nativo, como también en cada punto seleccionado del área invadida por $U$. molinae, se realizó el levantamiento del total de la flora vascular en unidades muestrales rectangulares de $10 \mathrm{~m}^{2}(5$ x $2 \mathrm{~m}$ ), mientras que para el bosque remanente se empleó un rectángulo de $500 \mathrm{~m}^{2}(25$ x $20 \mathrm{~m})$. Cada unidad se recorrió internamente de manera zigzagueante hasta cubrir la totalidad del área muestreada, registrando las especies de plantas mediante la aplicación del Método BraunBlanquet (Mueller-Dombois \& Ellenberg 1974), asignando la participación de cada una de las especies en categorías de cobertura de la vegetación. Esta cobertura (\%) se estimó por cada clase de altura según los estratos usados por CONAFCONAMA-BIRF (1999), con umbrales de 0,5, 1, 2, 4, 8, 12 y $20 \mathrm{~m}$. Se generó un valor de importancia (\%) para cada especie, el que se obtuvo a partir de la cobertura estimada en porcentaje, en cada parcela y separadamente para cada clase de altura. Se consideró una ponderación doble para los valores de cada clase de altura mayor, respecto de cada clase de altura precedente. De tal forma, se obtuvo luego un valor en porcentaje de cada especie en cada parcela, el cual se llevó a una proporción máxima de $100 \%$, para finalmente poder comparar entre especies y entre parcelas.

La Tabla 1 muestra la lista de las 16 especies encontradas, siguiendo el tratamiento taxonómico de Rodríguez et al. (2018), clasificando su participación relativa en valor de importancia tanto para el bosque fernandeziano como para los parches invadidos. Adicionalmente, se presenta la frecuencia de cada especie presente en los parches invadidos por $U$. molinae. La dispersión de los valores de la importancia relativa de cada especie dentro de los parches invadidos se aprecia en el gráfico de la Fig. 2. En tanto, la Fig. 3 muestra esquemas de la estructura vertical para cada tipo de unidad estudiada.

$U$. molinae presentó un valor de importancia promedio de 92,6\% en los parches invadidos. Otras especies alóctonas registradas fueron Aristotelia chilensis y Rubus ulmifolius, pero en comparación al amplio dominio de $U$. molinae, se observaron con una importancia mucho menor (ver Tabla 1).

La principal especie nativa de planta vascular observada como capaz de sobrevivir en estos parches invadidos correspondió al arbusto ericáceo Gaultheria racemulosa, endémico de Juan Fernández y propio del matorral montano (Teillier \& Escobar 2013). Éste presentó una frecuencia de $100 \%$ en los parches de $U$. molinae y corresponde a la especie nativa y endémica con el valor de importancia más elevado en estos parches invadidos evaluados. Resulta relevante notar que G. racemulosa posea el mismo hábito de crecimiento y similar requerimiento de hábitat de zonas mésicas y expuestas que la invasora y que sea capaz de sobrevivir a ella. Este hallazgo destaca el rol potencial de este arbusto ante eventuales estrategias de control de la

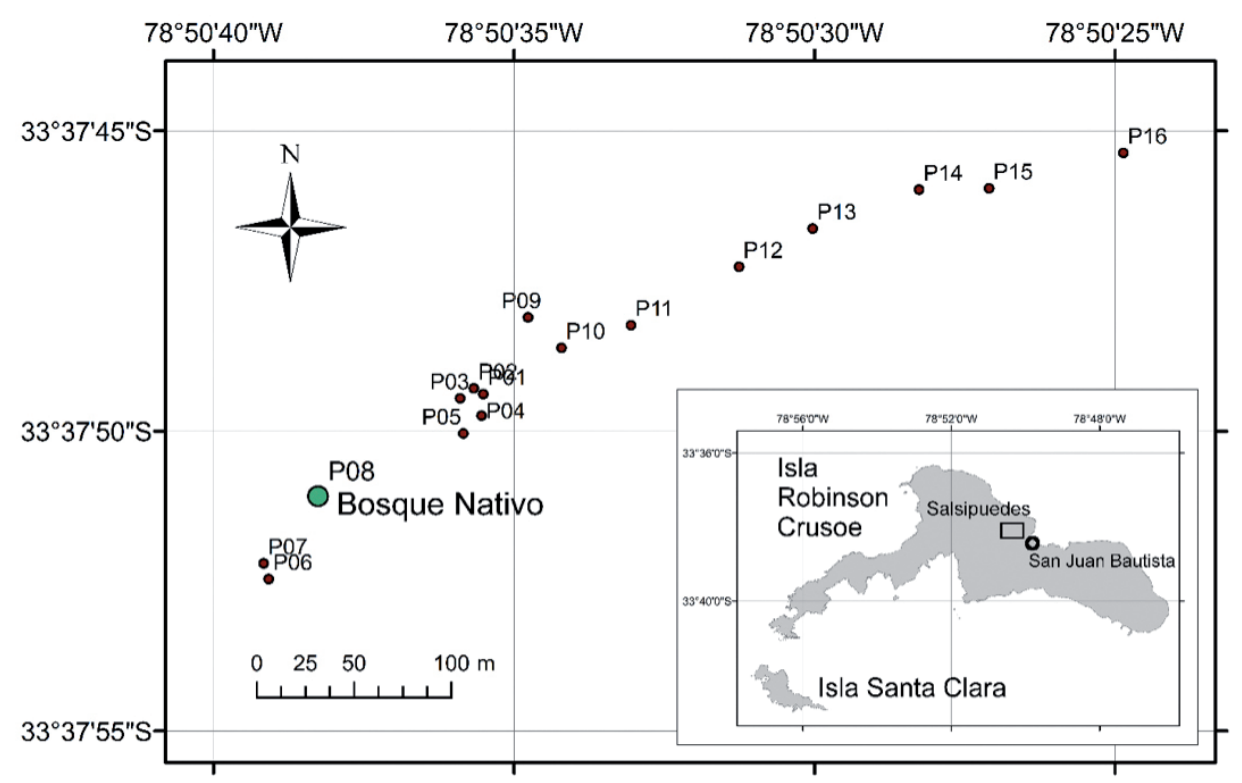

Figura 1. Mapa de localización de los puntos muestreados en sector Salsipuedes, Isla Robinson Crusoe. / Location map of the sampled points in Salsipuedes area, Robinson Crusoe Island. 
invasora, permitiendo aventurar que un control mecánico de $U$. molinae que procure dejar los ejemplares de $G$. racemulosa creciendo en estos parches, podría permitir a esta última especie acrecentar su dominio en estos parches en el futuro, con una mayor capacidad de albergar especies nativas. Otros arbustos nativos y endémicos con alta frecuencia (40\%), aunque con bajos valores de importancia, fueron Erigeron fernandezius, especie frecuente en sitios alterados como las orillas de senderos, y Escallonia callcottiae que, aunque más escasa, se encuentra en lugares abiertos y expuestos.

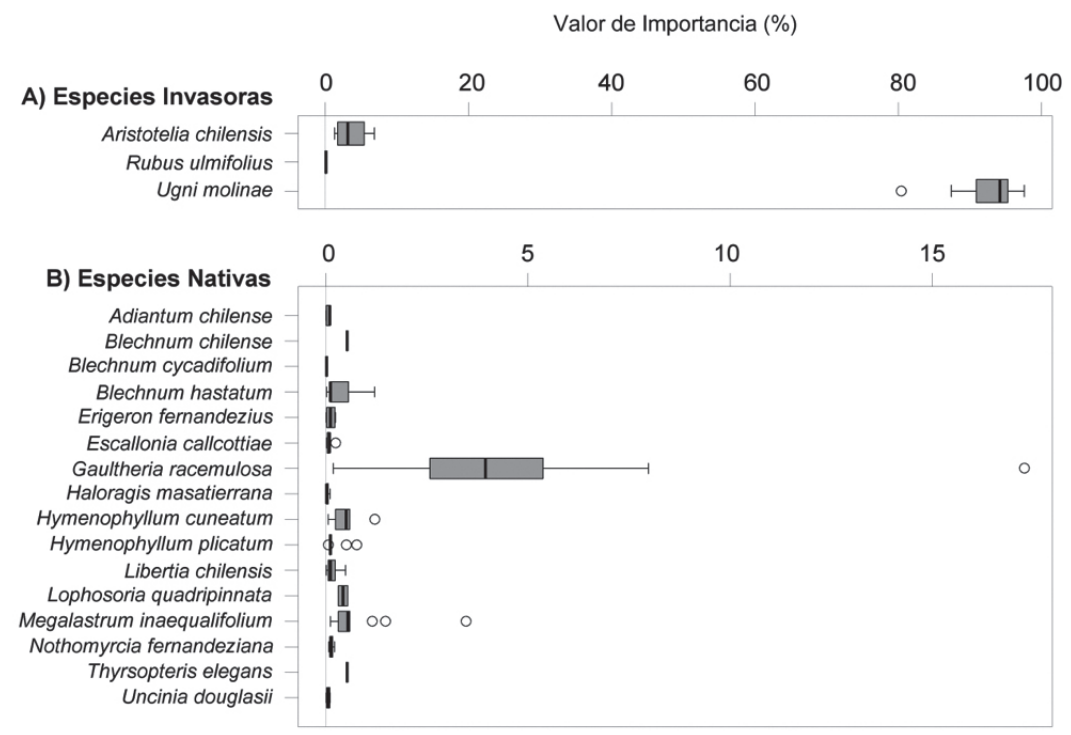

Figura 2. Gráfico comparativo de la dispersión de los valores de importancia de las plantas (A) invasoras y (B) nativas, presentes en los parches invadidos por U. molinae. / Comparative graph of the dispersion of the importance values of (A) invasive plants and (B) native plants, both present in the U. molinae-invaded patches.
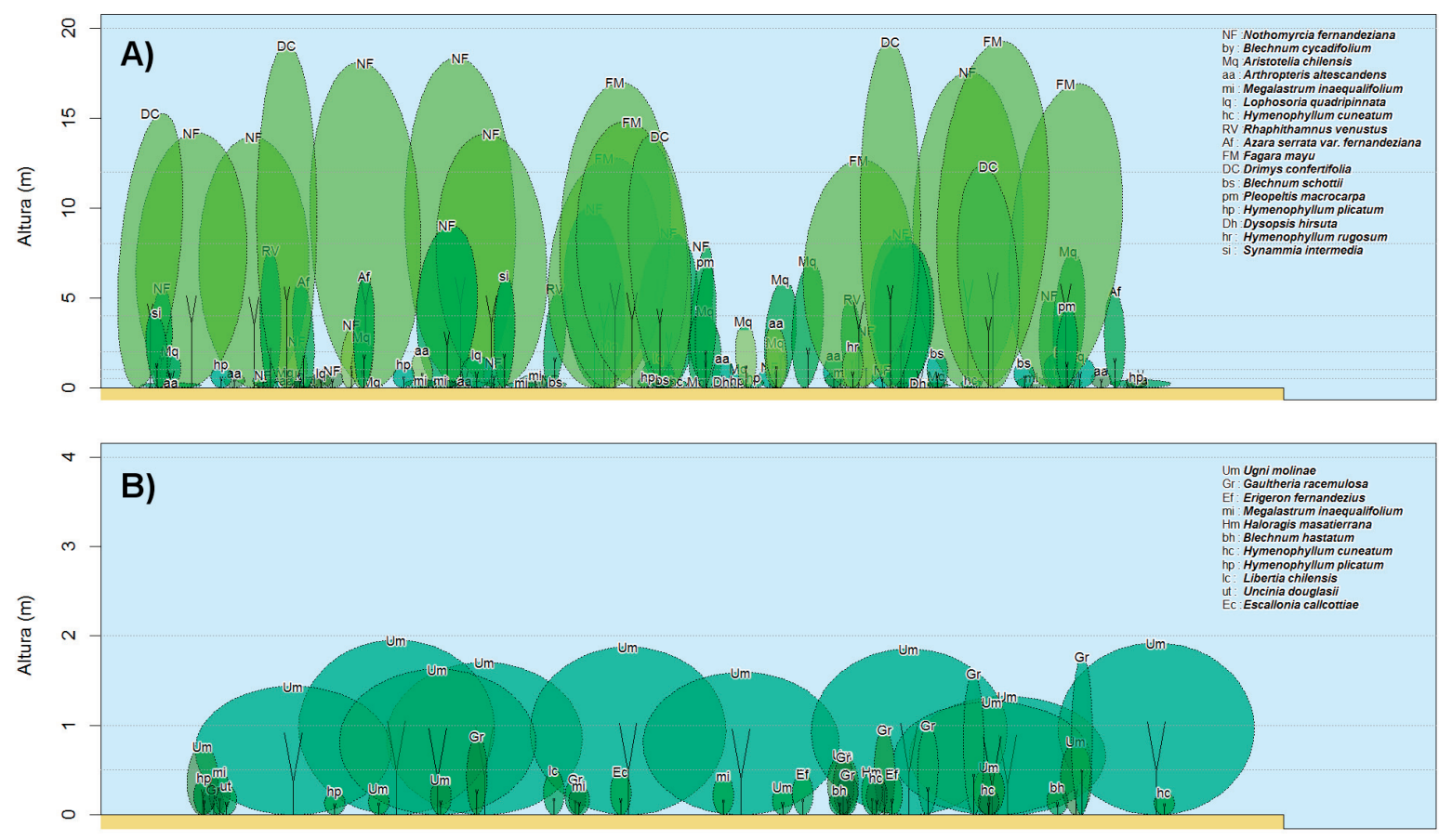

Figura 3. Esquema de estructura vertical de las comunidades analizadas: (A) Bosque remanente fernandeziano y (B) Parche número 11, invadido por U. molinae. / Vertical structure diagram of the analyzed communities: (A) Fernandezian relict forest and (B) U. molinaeinvaded patch number 11 . 
Plantas nativas bajo invasión de Ugni molinae en Robinson Crusoe: Alarcón, D. ET AL.

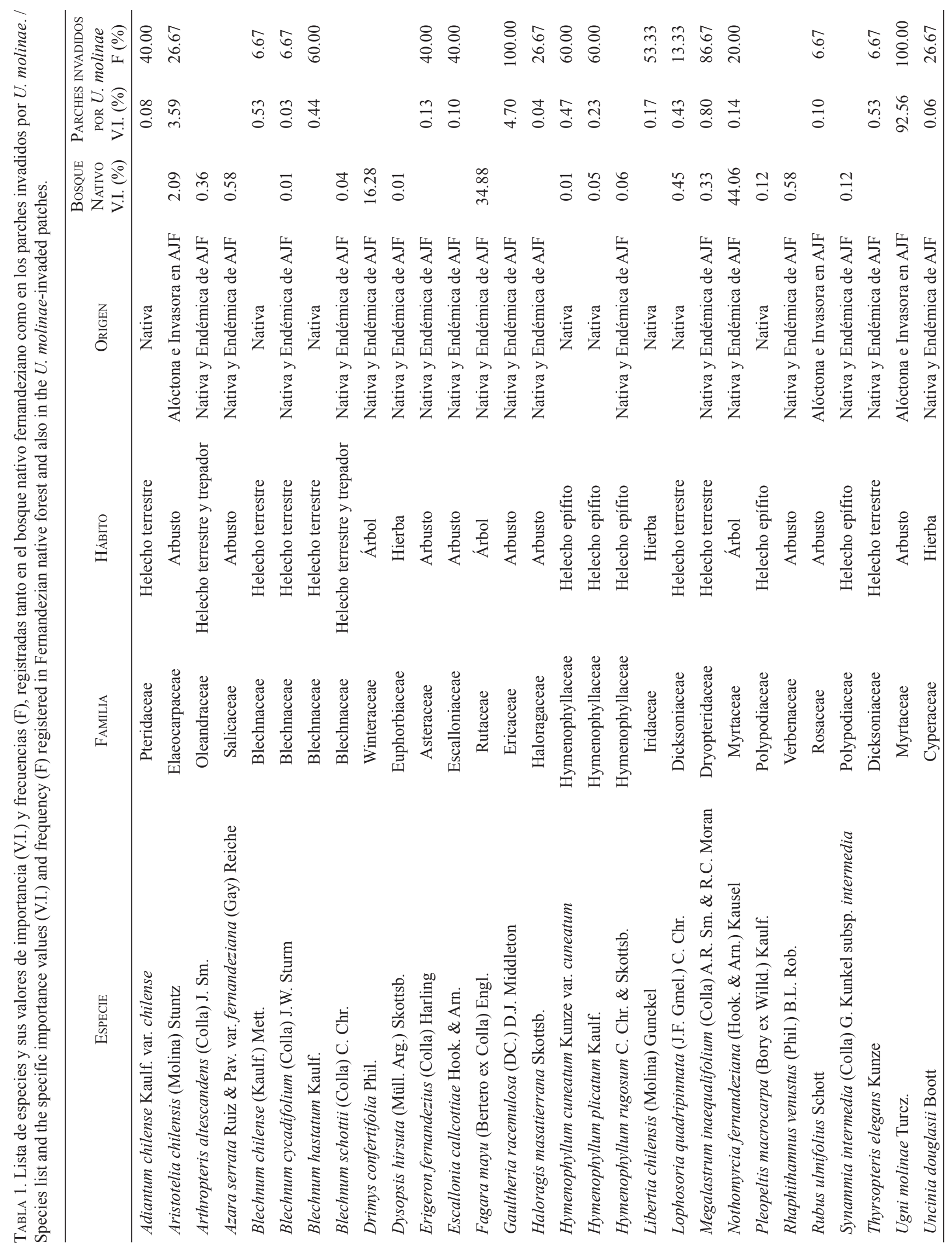


Otras especies relevantes dentro de las encontradas capaces de vivir en los parches invadidos incluyeron helechos terrestres con frecuencia heterogénea, como Megalastrum inaequifolium (87\%), Lophosoria quadripinnata (13\%), Thyrsopteris elegans $(7 \%)$ y tres especies del género Blechnum, siendo B. hastatum la más frecuente $(60 \%)$. Cabe señalar que los helechos terrestres han sido propuestos como posibles plantas nodrizas de especies del bosque nativo fernandeziano (Bastías 2010). Algunas especies de helechos epífitos (Hymenophyllum cuneatum e $H$. plicatum) se presentaron en una frecuencia alta $(60 \%)$ en la base de los arbustos de $U$. molinae y $G$. racemulosa. Fuera del área muestreada, se pudo observar a una plántula del árbol Drimys confertifolia, que se puede encontrar también como arbusto en los márgenes de vegetación baja, se pudo observar regenerando entre $U$. molinae. Sin embargo, la única especie arbórea endémica regenerando en las parcelas muestreadas invadidas fue Nothomyrcia fernandeziana, la cual pese a haber sido registrada con una baja frecuencia $(20 \%)$, se trata de un árbol relevante en los bosques montanos y de altura.

Se observó una relación inversa $(\mathrm{p}=0,048)$ entre un índice de la distancia entre cada parche dominado por $U$. molinae y el bosque fernandeziano remanente (expresado como la raíz cuadrada de la distancia en metros), y la riqueza de especies nativas en cada punto, de acuerdo al modelo lineal graficado en la Fig. 4. Esto indicó que mientras más

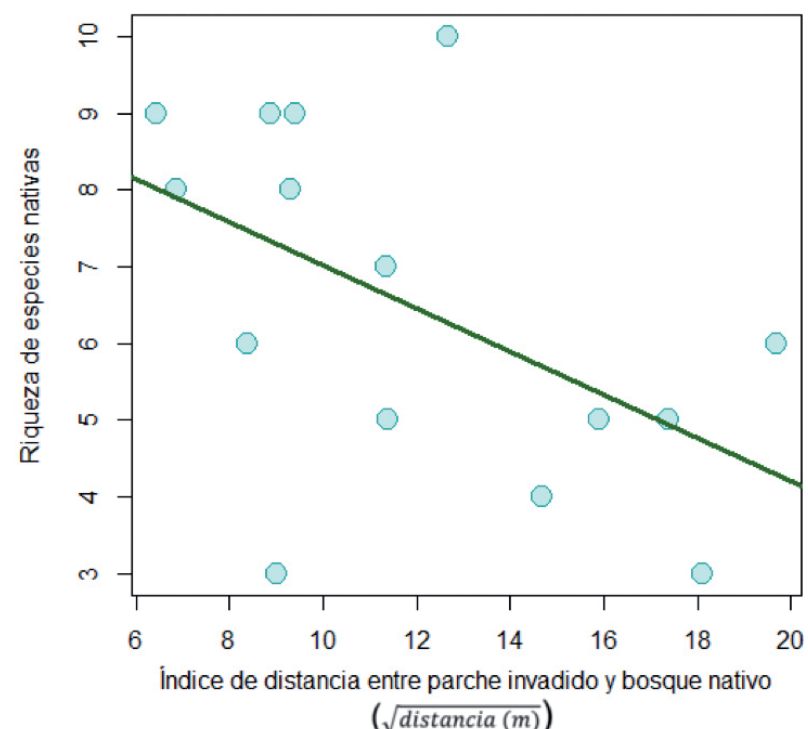

FIGURA 4. Gráfico de la relación entre la riqueza de especies nativas presentes en los parches invadidos por $U$. molinae y el índice de distancia entre dichos parches y el bosque nativo remanente. / Graph of the relationship between the native species richness found in the $U$. molinae-invaded patches and the index of distance between invaded patches and the relict native forest. cerca estén los parches invadidos al bosque fernandeziano, presentan mayor riqueza de especies nativas, relevando así la importancia de estos relictos de bosque, respecto de su potencial para recolonizar con ejemplares de especies nativas.

Se observó una relación directa a través de un modelo lineal $(p=0,033)$ entre la riqueza de especies nativas en los parches invadidos y la altitud de dichos parches. Esto implica que mientras más alto el parche invadido, mayor riqueza de plantas nativas, como se aprecia en la Fig. 5. Ya que el parche de bosque nativo remanente se halla en la porción más alta del área estudiada (490 m s.n.m.), se constató una muy alta correlación negativa $(\mathrm{r}=-0,97)$ entre la altitud y la distancia de los parches al bosque nativo remanente. Lo anterior puede indicar que la riqueza de especies nativas convivientes con la invasora sería dependiente de la distancia al parche remanente, pero de igual modo relacionado con la altitud de la zona invadida. Dado que es posible asumir que la invasión de U. molinae se inició probablemente desde el poblado, es decir desde altitudes menores, esto podría ser indicio que puntos con mayor tiempo de invasión, asociados a altitudes menores, podrían conservar un menor número de especies nativas que aquellos parches de invasión eventualmente más recientes (a altitudes mayores) y más cerca de los parches nativos remanentes que puedan actuar como fuente de propágulos de plantas nativas y endémicas.

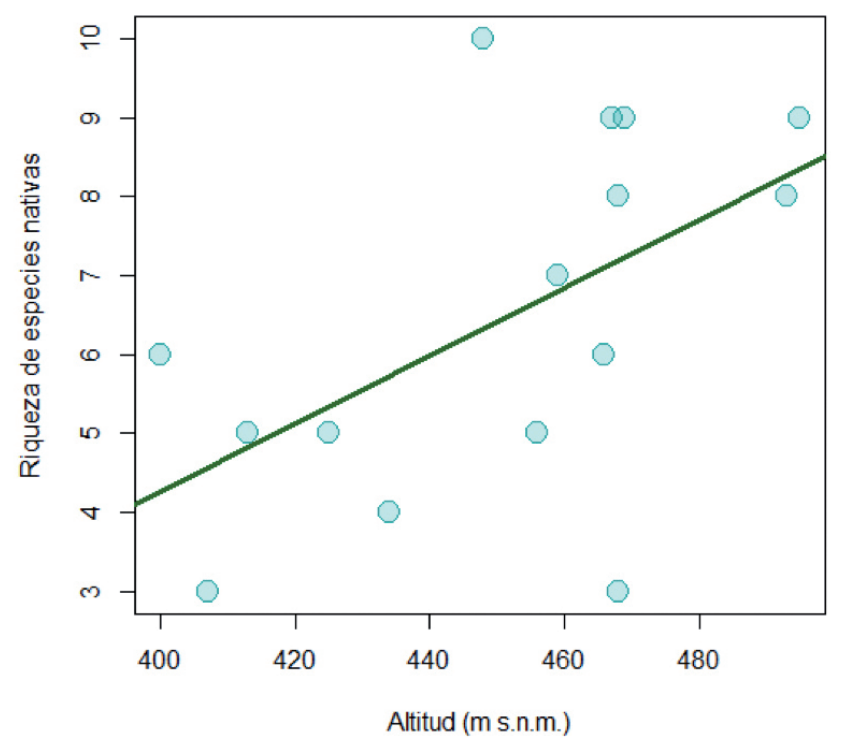

FIGURA 5. Gráfico de la relación entre la riqueza de especies nativas presentes en los parches invadidos por $U$. molinae y la altitud de dichos parches. / Graph of the relationship between native species richness found in $U$. molinae-invaded patches and its elevation above sea level. 


\section{AGRADECIMIENTOS}

A la Corporación Nacional Forestal (CONAF) y en especial a Iván Leiva, Felipe Sáez, Ramón Schiller, Alfonso Andaur, Bernardo López, Guillermo Araya, Maximiliano Recabarren y Danilo Arredondo, del Parque Nacional Archipiélago de Juan Fernández, por las facilidades de acceso y apoyo en terreno. Al proyecto Fondecyt 1160794.

\section{REFERENCIAS}

BAstíAs, I. 2010. Los helechos en la sucesión secundaria de bosques en la isla Robinson Crusoe y su potencial en restauración ecológica. En: IEB. Conservación y restauración ecológica de las islas de Juan Fernández. Informe Avance de investigaciones. p.11.

Bastías, I. 2014. Desarrollo de una metodología para el control mecánico y químico de Ugni molinae (Murta) en la isla Robinson Crusoe, Archipiélago de Juan Fernández. Informe de Consultoría para el Proyecto GEF №83266. URL: https://docplayer.es/6831992-Desarrollo-de-unametodologia-para-el-control-mecanico-y-quimico-de-ugnimolinae-murta-en-la-isla-robinson-crusoe-archipielagojuan-fernandez.html. Accedido: Octubre 25, 2017.

CONAF-CONAMA-BIRF. 1999. Catastro y Evaluación de los Recursos Vegetacionales Nativos de Chile. Informe Nacional con Variables Ambientales. Santiago. 88 pp.

Danton, P., Perrier C., Martínez de Reyes, G. 2006. Nouveau catalogue de la flore vasculaire de l'archipel Juan Fernández (Chili). Acta Botanica Gallica 153(4): 399-587.

DíAz, R. 2012. Análisis y modelación de la evolución espaciotemporal de la invasión de Rubus ulmifolius, Aristotelia chilensis y Ugni molinae, en la Isla Robinson Crusoe. Universidad de Chile, Tesis Facultad de Ciencias Forestales y de la Conservación de la Naturaleza, Santiago, Chile. 55 pp.

Dirnböck, T., Greimler, J., López-Sepúlveda, P., Stuessy, T.F. 2003. Predicting future threats to the native vegetation of Robinson Crusoe Island, Juan Fernandez Archipelago, Chile. Conservation Biology 17(6): 1650-1659.
Funk, J.L., Vitousek, P.M. 2007. Resource-use efficiency and plant invasion in low-resource systems. Nature 446: 1079-1081.

Greimler, J., Stuessy, T.F., Swenson, U., Baeza, C.M., Matthei, O. 2002. Plant invasions on an oceanic archipelago. Biological Invasions 4:73-85.

Johow, F. 1896. Estudios sobre la flora de las islas de Juan Fernández. Imprenta Cervantes, Santiago, Chile. 288 pp.

KaWAKami, K., Окосні, I. 2010. Restoring the Oceanic Island Ecosystem. Springer Japan, Tokyo. 216 pp.

Lockwood, J.L., Hoopes, M.F., Marchetti, M.P. 2013. Invasion Ecology. John Wiley \& Sons, West Sussex, UK. 304 pp.

Mueller-Dombois, D., Ellenberg, H. 1974. Aims and methods of vegetation ecology. John Wiley \& Sons, Nueva York. 547 pp.

Rodríguez, R., Marticorena, C., Alarcón, D., Baeza, C., Cavieres, L., Finot, V.L., Fuentes, N., Kiessling, A., Minoc, M., Pauchard, A., Ruiz, E., Sánchez, P., Marticorena, A. 2018. Catálogo de las plantas vasculares de Chile. Gayana Botanica 75(1): 1-430.

Smith-Ramírez, C., Arellano, G., Hagen, E., Vargas, R., Castillo, J., Miranda, A. 2013. El rol de Turdus falcklandii (Aves: Passeriforme) como dispersor de plantas invasoras en el archipiélago de Juan Fernández. Revista Chilena de Historia Natural 86(1): 33-48.

Smith-Ramírez, C., Vargas, R., Castillo, J., Mora, J.P., ArellanoCataldo, G. 2017. Woody plant invasions and restoration on forests of island ecosystems: lessons from the Robinson Crusoe Island. Biodiversity and Conservation 26(7): 15071524.

Stuessy, T.F., Marticorena, C., Swenson, U., Greimler, J., LóPEZ-SEPÚlVEDA, P. 2018a. Impacts on the Vegetation. In: Stuessy, T.F., Crawford, D.J., López-Sepúlveda, P., Baeza, C.M., Ruiz, E. (eds.) Plants of Oceanic Island. Pp: 117-133. Cambridge University Press, New York, USA.

Stuessy, T.F., Rodríguez, R., Baeza, C.M., López-Sepúlveda, P. 2018b. Taxonomic Inventory. In: Stuessy, T.F., Crawford, D.J., López-Sepúlveda, P., Baeza, C.M., Ruiz, E. (eds.). Plants of Oceanic Island. Pp: 57-97. Cambridge University Press, New York, USA.

TeIllier, S., Escobar, F. 2013. Revisión del género Gaultheria L. (Ericaceae) en Chile. Gayana Botanica 70(1):136-153.

Recibido: 10.11 .2017

Aceptado: 04.03.2019 